\title{
A Patrol Grid Protocol for Mobile Wireless Sensor Network
}

\author{
Dongliang Xie \\ Mingxing Chen \\ State Key Laboratory of Networking State Key Laboratory of Networking \\ \& Switching Technology \\ Beijing University of Posts \& \\ \& Switching Technology \\ Beijing University of Posts \& \\ Telecommunications, Beijing, China Telecommunications, Beijing, China \\ xiedl@bupt.edu.cn \\ today241@sina.com
}

\author{
Chen Canfeng \\ Nokia Research Center \\ Beijing, China \\ canfeng-david.chen@ \\ nokia.com \\ Jian Ma \\ Nokia Research Center, \\ Beijing, China \\ Jian.J.Ma@nokia.com
}

\begin{abstract}
Nowadays more requirements for wireless sensor networks are utilized to military, science and our daily life gradually, however the sink mobility is still bottleneck problem in a number of WSN application scenarios. In some fields like environmental monitoring, the solution is an especially urgent request. In this paper we propose Patrol Grid Protocol (PGP), a sink mobility supported route protocol for environmental monitoring which builds on a previously developed routing protocol called TWOTier Data Dissemination (TTDD). We prove that PGP can be more suitable for urgent events and query-driven mode than TTDD. The description of mechanisms and simulation are presented firstly, then we evaluate the performance of the new protocol and analyze the overhead and delay. PGP is a more applicable protocol for environmental monitoring.
\end{abstract}

\section{Categories and Subject Descriptors}

C2.2 [COMPUTER-COMMUNICATION NETWORKS]: Network protocols. C4 [PERFORMANCE OF SYSTEMS]: Performance attributes. J2 [COMPUTER APPLICATIONS]: Physical science and engineering.

\section{General Terms}

Design, Performance, Experimentation.

\section{Keywords}

Wireless sensor networks, TTDD, Performance, Environmental monitoring.

\section{INTRODUCTION}

A wireless sensor network (WSN) consists of a large number of micro sensing nodes by means of wireless transmissions, deployed in an area of interest. It could provide data at granularities unrealizable by other means. Compared with the traditional networks, the main target of the WSN routing protocols is the assurance of report messages delivered to the remote base station (BS or sink node) instead of a kind of transmission supply (e.g. P2P). Then BS aggregates and analyzes the report messages received and decides whether there is an unusual or concerned event occurrence in the area of interest [1]

Because wireless sensor networks are those in which nodes are low-cost sensors that can communicate with each other in a WICON'08, November 17-19, 2008, Maui, Hawaii, USA.

Copyright 2008 ICST 978-963-9799-36-3. wireless manner, have limited computing capability, and memory and operate with limited battery power. Sensors are energyconstrained and difficult to recharge. While designing the protocols, energy consumption is the first one to be considered. In recent years, more researches are focused on the mobility of the sink, for Mobile sink can cause the balance of energy while roaming about the network. There are a lot of routing protocols which are designed specifically according to the characteristic of the wireless sensor networks with mobile sink(s). Also, the WSN routing protocols are always associated with the applications tightly. As one kind of the most important typical applications, environmental monitoring is taken into our consideration. Several sinks are taken by moving inspectors in a region of interest. Once an interest appears, sensing data would be generated and sent to sinks by multi-hop forwarding. In this paper we use the key idea of Two-Tier Data Dissemination (TTDD) protocol [2] and propose PGP (Patrol Grid Protocol).

The main procedures of PGP include: 1) grid construction, a twotier grid structure divides the sensor field into many cells and the grid points take charge for data transmission; 2) hierarchicalmonitoring, a mechanism for event detecting timely and trend forecast; 3) query-driven, it offers a method for user acquiring information actively; 4) sink mobility, supported by three stationary sensor nodes as sink agents; 5) grid maintenance.

The remainder of the paper is organized as follows: Section 2 provides a brief introduction of several related works. Section 3 describes details of PGP. Section 4 includes a comparative analysis of theoretical overhead for TTDD and PGP. Section 5 gives performance evaluation results. At last we conclude this paper.

\section{RELATED WORK}

Many researches have been done with the mobility of the sink in WSN [3]. Because stationary sink can bring a lot of drawbacks such as the heavy load of the nodes which are near to the sink, many researchers proposed to deploy mobile sink into WSN. This can prevent the appearances of the bottleneck nodes, balance the load of each node, and extend the whole lifetime of the networks. However, along with the new proposal, sink mobility brings new challenge into the WSN routing protocols design. All the common nodes are stationary, location-aware and addressed by a unique identifier after they deployed. With responsibility to collect sensing data, WSN need to get moving sinks' location anytime. When the sinks location changed, the location updates to the whole network could cause many problems, for instances, 
excessive drain of sensors' limited battery power supply and increased collisions in wireless transmissions. Some methods have been explored on the base of geo-based routing protocols. In neighbor-casting update method, a mobile sink periodically sends its location to its one-hop neighbors, but the neighbors don't forward the location update message further. Under this circumstance, most of the nodes have to send data to an outdated sink location and a longer forwarding route may be resulted causing more energy consumption. Some proposed location service required the senders to query the up-to-date location of the sink before data sending, e.g. LAR[4],GLS[5] and SLALoM[6] etc. others let the mobile sink actively send the location update message to the sensor nodes such as DREAM[7], and GPSR[8]. But always sending location update messages to multiple sensor nodes need to maintain a multicast group maybe a heavy load to the network.

Several protocols were presented to solve sink mobility supported problem such as TTDD, SEAD [9], and EARM [10]. We made a deep study of TTDD which is considered as a feasible protocol for this kind of application.

In TTDD, a grid structure which divides the sensor field into many cells is constructed by a source proactively propagates the existent sensing data globally [11]. Then the sensors on the grid cross-points will serve as dissemination nodes (DN) and store the data. When a sink query is flooded within a local gird cell, the DNs are responsible for forwarding it upstream to data sources along specific grid branch and pulling sensing data downstream toward the sink.

TTDD is applicable for the event-driven case [12]. In this situation, however, sinks must flood queries for the sensing data periodically. TTDD doesn't fit for the outburst because when the interest appears, it couldn't push the information to sink immediately. TTDD isn't suitable for the query-driven application either. As we considered, most of the environment incidents are paroxysmal and we often need to get the monitoring data initiatively. When a number of sensors detect the interesting, each of them will build a grid in the whole network. It takes redundant overhead to the WSN. Therefore we propose PGP, a more applicable protocol for the environmental monitoring.

\section{PGP}

In PGP, only one of the deployed sinks starts to construct a twotier grid, a bottom finely granular grid and a top coarsely granular grid. The bottom cells are $\alpha \times \alpha$ squares. And each of the top cells is made of 4 bottom cells. We take the bottom and the top cross-points as GP0 and GP1. All of them are DNs and any of them could communicate with others by multi-hop.

Based on the two-tier grid, we present a hierarchical-monitoring mechanism which can inspect the region of interest effectively. At the same time, PGP supports query-driven applications and sink mobility. The content as follow will introduce the grid construction (3.1), hierarchical-monitoring mechanism (3.2), query-driven (3.3), sink mobility (3.4) and grid maintenance (3.5) in detail.

\subsection{Grid Construction}

The grid construction is the key step of PGP. For the sake of clearly description, we assume only one sink in the interest plane.
After the deployment of the sensors, everyone gets the information of its local neighborhood. Sink chooses one neighbor sensor as the first grid node called BaseGP. This node is also the GP1 which starts the construction of the two-tier grid. The process is as same as that in TTDD: BaseGP calculates the locations of its four neighboring dissemination points given its location and cell size $\alpha$. It propagates a grid build message taken the BaseGP location to the neighbor DPs using simple greedy geographical forwarding. Upon receiving the message, the closest node to each DP sends backward a message to the point from which the message was received and forwards the message to its other three adjacent DPs. It also stores the BaseGP location and promotes itself as a DN. These actions are repeated until the grid build message propagates throughout the whole network. Then all the chosen DNs could get the BaseGP and their neighbor DNs location. According to the distance to the BaseGP, the DNs classify themselves as GP0 or GP1. The main components of the two-tier grid are shown in Figure 1.

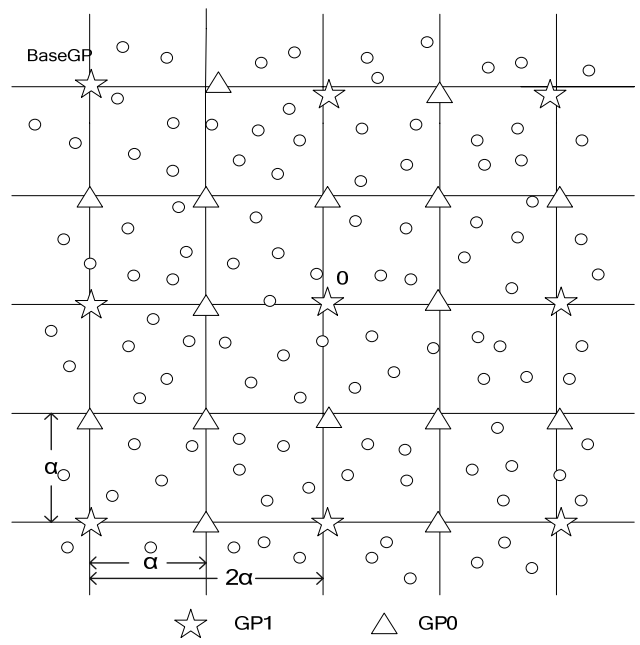

Figure 1. Two-tier grid in a PGP network

For sink mobility and hierarchical-monitoring, this sink chooses GA from all the neighbor sensors before the grid construction. The GA location is also sent to all the DNs by the grid build message.

\subsection{Hierarchical-monitoring}

After the grid construction, GP1s begin the coarsely granular monitoring. Each GP1 is a center spot of a $2 \alpha \times 2 \alpha$ square within which it takes charge of monitoring. It broadcasts its location and ID information within this field. Once the interest occurs, the sensor which discovers that sends an event message to the GP1. This GP1 promotes itself as an Event Source (ES) and steps in the finely granular monitoring with its eight neighbor GP0s. All the nine DNs collect the sensing data of the each $\alpha \times \alpha$ region centered by each of them.

If a GP1 senses an interest appears, it actuates the finely granular monitoring by constructing a tree which roots at itself (shown in Figure 2). And then each of the finely granular monitoring nodes (GP1 and GP0s) build a secondary monitoring tree according to 
the number of hops from the sensors in the monitoring field. That means there are nine secondary trees and one monitoring tree in a $3 \alpha \times 3 \alpha$ square. The sensing data which collect by the secondary trees will aggregate at the finely granular monitoring nodes and forward to the ES along the monitoring tree. If the ES hasn't received any event messages in a fix period of time set by the application, it should cancel the monitoring trees and the finely granular monitoring.

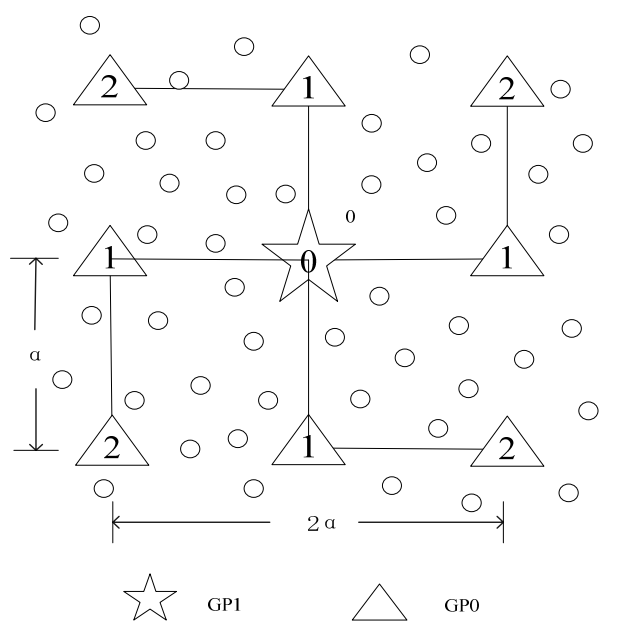

Figure 2. Monitoring Tree

With the hierarchical-monitoring as described above, there are two kinds of sensing data: the event data obtained from the coarsely granular monitoring and the sensing data gained by the finely granular monitoring. Finally, both of them are sent to the ES and transmitted to sinks.

When the GP1 receives the event data for the first time, no path exists between this ES and sink. The ES transmits the data to the Global Agent (GA) at first. Then the GA will deliver it to sink via the Primary Agent (PA) and Immediate Agent (IM).The path from sink to ES is built as follows: Firstly, sink finds an IM and a PM in its neighbors. Then it appoints a DN as its immediate dissemination node (IMDN) according to the ES location (obtained from the event message) and tells IMDN the PA information. This IMDN chooses the closest neighbor DN to the ES as the downstream DN. The action is seriatim until reaching the ES. As a result, the path is Sink -> IM -> PM -> IMDN -> downstream DN $->\ldots . .>$ ES. Each node on this path records the downstream and upstream sensors location/ ID. That means the reverse path from ES to sink comes into existence.

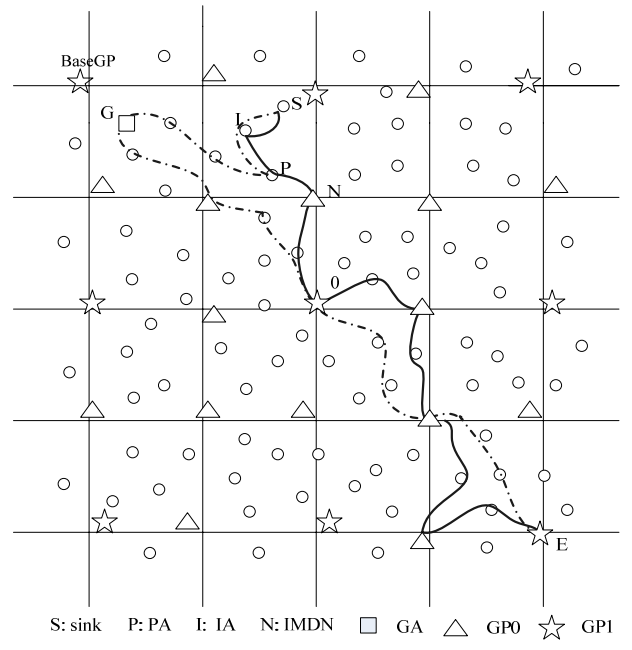

Figure 3. Setting up the event path

Figure 3 shows the process: the broken line represents the event data streams from the ES to sink by way of GA, PA and IA. The solid line shows the sink to ES path along which the monitoring data forwards.

\subsection{Query-driven}

In the environmental monitoring application, the query-driven may meets necessity of some conditions. For example, when monitoring forest for fire protection, the system should offer a possibility judgment function which needs the monitoring data at any minute. PGP query-driven mechanism can provides data initiatively. The command format and query target depend on the concrete application type. Here we only consider the application of forest fire monitoring. Based on the geographic position, the query is interested in some attribute (as the maximum, average, or minimum of humidity, temperature, light intensity) in a particular field.

If sink accepts a query command from the application layer, it stores the request and calculates the center of the query range (called virtual source VS). The VS is an ideal data source but not a real one. Then it is needed to find the closest sensor to the VS (called query source QS) to collect query data for sink. The seeking QS process is as same as the procedure of a query path built. Sink promotes a DN as an IMDN for the VS by its location and $\alpha$. The IMDN transmits path build message to the QS along the grid line using simple greedy geographical forwarding. This path is called query path. When the real source receives this message, it responds to sink along the query path. When the QS location and ID taken by the respond message is received by sink, sink send a query message to the QS which triggers it to collect the query data for sink. The process is shown as Figure 4. 


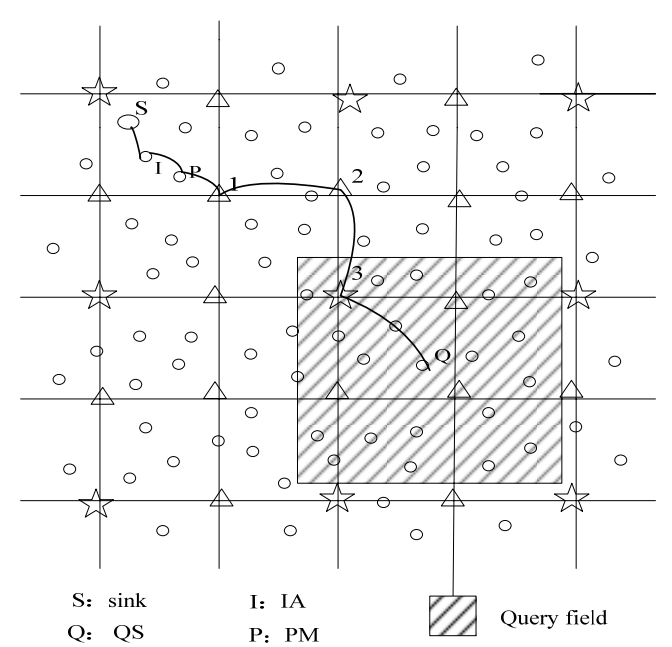

Figure 4. Query Path

\subsection{Sink Mobility}

This section describes the method how sink could receive data from sources (ESs and/or QSs) continuously. PGP divides the WSN into a grid of cells and uses these cells to locate moving sink. Sink has two agents in the cell within which it stays, an Immediate Agent (IA, one hop away from sink) and a Primary Agent (PA, represent sink in a cell to connect with the IMDNs). For IMDNs, the PA is on behalf of sink. And for PA, the IA which could communicate with sink directly is in sink's behalf. If an event happens, the GA is the sink representation for ES.

When sink moves out of the one-hop transmission range of its current IA, it picks a new IA from its neighboring nodes. Sink then sends an update to its PA and old IA to relay data. PA is not changed as long as the distance to sink less than $\alpha$. When some sources (ESs and/or QSs) exist in the region, sink need to build new paths to these sources after moving out of a cell. That means sink will change new IMDNs meanwhile it chooses new PA and IA.

As the difference of sources, the paths classified as event path and query path. PGP is multi-path supported. And the DNs in multi path must store the query ID or event ID which represent different paths. A query IMDN may be an intermediate DN in event path at the same time.

\subsection{Grid Maintenance}

At the hand of short-range radio, the grid build message may get lost and the grid becomes incomplete. For the purpose of avoiding this situation, PGP brings a period of grid patch. A timer (wait neighbor DN response) is set after the grid build message forwarding. When the expiration of this timer happens, DN checks the neighbor DN information stored in cache and sends grid build messages to those adjacent DPs which don't find corresponding neighbor DNs.

In order to prevent the grid points broken down by power consumption associated with the DN role, DN must find a neighbor sensor instead of itself. DN sends the grid point information to all of its neighbors after the grid patch. When DN becomes dead node, one of these neighbors promotes itself as the substituent and operates as the grid point.

\section{OVERHEAD AND DELAY ANALYSIS}

In this section we analyze the efficiency and scalability of PGP compared with TTDD. We measure two metrics: the communication overhead (set up paths) and the delay. Because TTDD is event-driven protocol, we only compare the event data collection situation.

We consider that a square sensor field of area $\mathrm{A}$ in which $\mathrm{N}$ sensor nodes are uniformly distributed so that on each side there are approximately $\sqrt{N}$ sensor nodes. There are one sink in the sensor field. It traverses $\mathrm{M}$ cells at an average speed $\mathrm{v}$, while receiving data packets from $\mathrm{K}$ source. Each data packet (both event and monitoring) has a unit size $l$.

In PGP, the communication overhead is originated by grid construction and paths building between sink and the K sources. The sensor field is divided into cells; each has an area $\alpha^{2}$. Then the number of sensor nodes in each cell is $\mathrm{n}=\frac{N \alpha^{2}}{A}$ and $\sqrt{n}$ sensor nodes on each side of a cell. There are $\frac{N}{n}$ cells in the grid. The overhead for grid construction is

$$
\frac{4 N \sqrt{n}}{n} l=\frac{4 N}{\sqrt{n}} l
$$

The communication overhead for paths setting includes ES forwarding data to sink via GA and sink to ES along the grid line. We first analyze the worst-case communication overhead of ES to sink. We assume ES and GA locate at the vertexes of grid diagonal and sink is situated at another vertex of grid. The overhead of data transmission from ES to GA is $l \sqrt{2 N}$ and that from GA to sink is about $l \sqrt{N}$. Then the sum is $l \sqrt{N}(1+\sqrt{2})$. The overhead of data forwarding from sink to ES is $l \sqrt{2 N}$. For $\mathrm{k}$ sources, the overhead of path setting is $k l \sqrt{N}(1+2 \sqrt{2})$. Plus the overhead $\frac{4 \mathrm{~N}}{\sqrt{\mathrm{n}} l}$ in constructing the grid, the total overhead of PGP becomes:

$$
C O_{\mathrm{PGP}}=\frac{4 N}{\sqrt{n}} l+k l \sqrt{N}(1+2 \sqrt{2})
$$

In TTDD, the communication overhead is originated by $\mathrm{k}$ grids construction, sink flooding queries and paths setting. Each grid build overhead is as same as PGP, $\frac{4 N}{\sqrt{n}} l$; the query flooding overhead is ${ }^{n l}$; the path setting overhead for one source is $l \sqrt{2 N}$. Since the total overhead of TTDD is:

$$
C O_{T T D D}=k l\left(\frac{4 N}{\sqrt{n}}+\sqrt{2 N}\right)
$$

To compare TTDD and PGP, we have:

$$
\frac{C O_{\mathrm{PGP}}}{C O_{\text {TTDD }}} \approx \frac{1}{k} \quad N>n
$$


Thus, in a large-scale sensor network, PGP communication overhead is lower compared with TTDD as the number of sources increases. And in actual fact, the source number is well beyond one in environmental monitoring application.

The averaged delay of the sink-source pairs after path build is much the same in these two route protocols. We discuss the first delay which is between the moment ES discover the interest and the moment sink receives the first event data. This is a key index of protocol performance which indicates if sink can perceive the event in time. It is related with the GA and sink location in PGP. We consider the worst-case: ES and GA locate at the vertexes of grid diagonal and sink is close to ES. The process is GA sends data to PA after it receive it from ES. Then PA transmits it to IA which could turn it to sink in one-hop. It depends on the simple greedy geographical forwarding. We assume one node relay time is t. As a result, the total time is:

$$
D_{\mathrm{PGP}}=2 t \sqrt{2 N}
$$

In TTDD, the event data is not pushed to sink in the wake of generation. Sink floods query within a cell square for data request. If the flooding meantime is $\mathrm{T}(\mathrm{T}>>t$, it is associated with sink average speed). For the worst-case, the distance between sink and ES is as long as possible in the grid. The delay is the sum of T1 (time between the interest is detected by source and forwarded to the nearest $\mathrm{DN}$ ) and $\mathrm{T}$. Therefore, the first delay of TTDD is:

$$
D_{T T D D}=t \sqrt{2 N}+T
$$

To compare the first delay of TTDD and PGP, we have:

$$
\frac{D_{\mathrm{PGP}}}{D_{T T D D}}=\frac{2 t \sqrt{2 N}}{t \sqrt{2 N}+T}
$$

As assumed, we can get ${ }^{T=\alpha / v}$. And $\alpha$ should double the radio radius $r=30 \mathrm{~m}$.If the speed of sink moving $v$ is the same as the maximum of car in highway; it will be $30 \mathrm{~m} / \mathrm{s}$. Then we get the minimum $\mathrm{T}=2 \mathrm{~s}$. With the experiment result, $\mathrm{t}$ is about $0.02 \mathrm{~s}$. That is, if $N<5000, D_{\mathrm{PGP}}<D_{\text {TTDD }}$. Overall PGP can discover the interest timely than TTDD in worst-case.

\section{PERFORMANCE EVALUATION}

In this section, we evaluate PGP performance through simulations. We describe our simulation methodology in Section 5.1. Section 5.2 shows PGP superiority compared with TTDD by the simulation results.

\subsection{Simulation Methodology}

To facilitate comparisons with TTDD, we use the same simulation scenarios for these two protocols in Tossim. TOSSIM is a discrete event simulator for TinyOS sensor networks. Instead of compiling a TinyOS application for a node, users can compile it into the TOSSIM framework which runs on a PC. It allows us to debug, test, and analyze protocols in a controlled and repeatable environment. And it is more convenient to apply the protocol in actual network.

We take three metrics to evaluate the performance: success rate, average delay and first delay. The success rate is the ratio of the number of successfully received reports at a sink to the total number of reports generated by a source, averaged over all source-sink pairs. It indicates the efficiency of the data delivery. The average delay is defined as delay in TTDD and measured separately from monitoring data. It shows the freshness of data packets. The first delay is the time from the moment ES discover the interest to the moment sink receives the first event data. It is very important for sensitivity judgment in this monitoring application.

The characteristics of simulation scenarios are assumed as:

- The radio radius range in the simulations is $30 \mathrm{~m}$

- Sensor nodes are deployed in a two dimensional area evenly and the distances of adjacent nodes are less than $27 \mathrm{~m}$ (slightly less than radio rang)

- The grid size $\alpha$ is $60 \mathrm{~m}$ (double radio rang) in PGP and TTDD

- The trajectory of sink is along the edge of the region

For the evaluation of protocols each metric is evaluated as a function of the network size, the speed of sink and the number of sources.

\subsection{Simulation Results}

\subsubsection{Network Scale}

We first study how network scale affects PGP. We change the network scale in this group of simulations and set the sink speed $3 \mathrm{~m} / \mathrm{s}$; only one source is in the interest field. We design 5 different size of the WSN: 50 nodes in $180 \times 180 \mathrm{~m}^{2}, 75$ nodes in $200 \times 250 \mathrm{~m}^{2}, 100$ nodes in $270 \times 270 \mathrm{~m}^{2}, 125$ nodes in $300 \times 320 \mathrm{~m}^{2}$ and 150 nodes in $320 \times 350 \mathrm{~m}^{2}$.

Figure 5 shows the success rate of the data retrieving with different network scales. As we can clearly see, the success rate of PGP is higher than that of TTDD.

While, Figure 6 shows the average delay that caused by TTDD compared with that by PGP. In this way, PGP works better than TTDD. In a word, PGP is affected by network scale less than TTDD.

Figure 7 shows the first delay as the network scale changes. There is an inflection point in TTDD. The reason of this is the time between the moment the query sent in any query flooding and the moment DNs around sink receive event data at the first time.

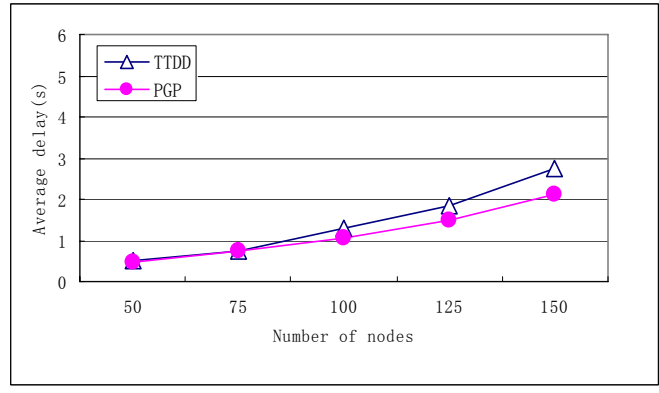

Figure 5. Success rate with different scale. 


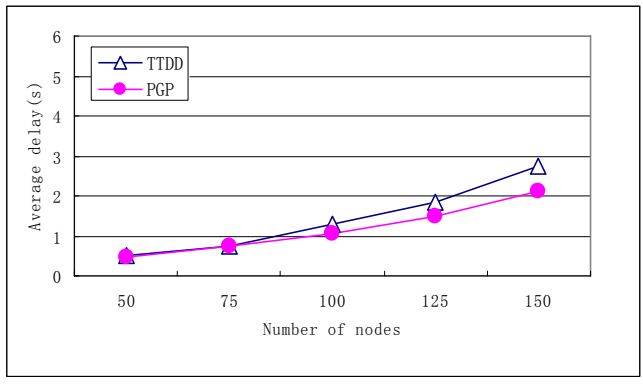

Figure 6. Average delay with different scale.

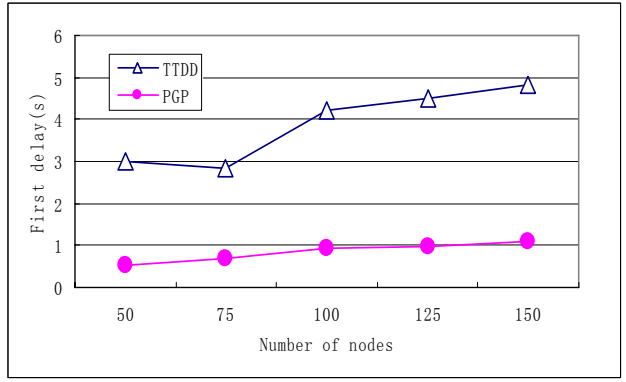

Figure 7. First delay with different scale.

\subsubsection{Sink speed}

In this section we evaluate how sink speed affects PGP. We distribute 100 sensor nodes in a $270 \times 270 \mathrm{~m}^{2}$ field and set one of them as event source. Sink speeds used are $0 \mathrm{~m} / \mathrm{s}, 3 \mathrm{~m} / \mathrm{s}, 6 \mathrm{~m} / \mathrm{s}$, $9 \mathrm{~m} / \mathrm{s}$ and $12 \mathrm{~m} / \mathrm{s}$ separately.

From figure 8, we can see when the speed of the sink is realtive low, the success rate is not so different between PGP and TTDD. With the speed increases, the success rate of PGP is improving better than that of TTDD.

In figure 9, we can also see that the average delay between PGP and TTDD has some distinctions as the speed of the sink increases. Better performance of averange delay can be aquired through PGP.

Figure 10 shows that the comparasion of the first delay through PGP with TTDD. We can clearly see that the first delay of TTDD is not so stable as that of PGP as the speed of the sink increases. it will not change the first delay much as the speed of the sink changes.

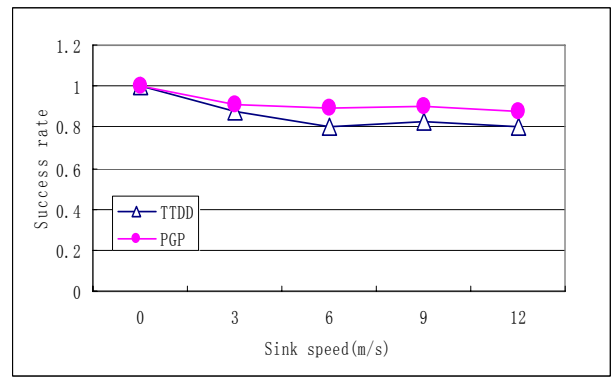

Figure 8. Success rate with different speeds.

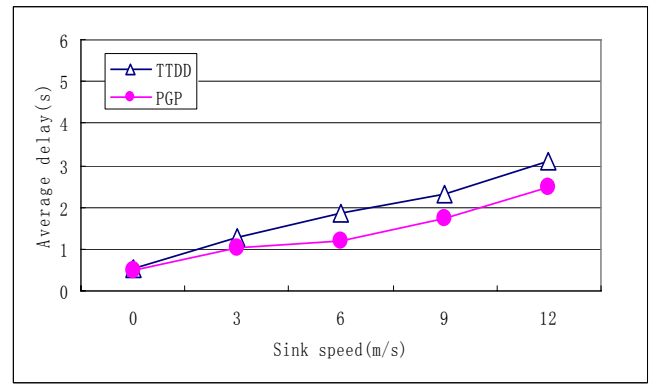

Figure 9. Average delay with different speeds.

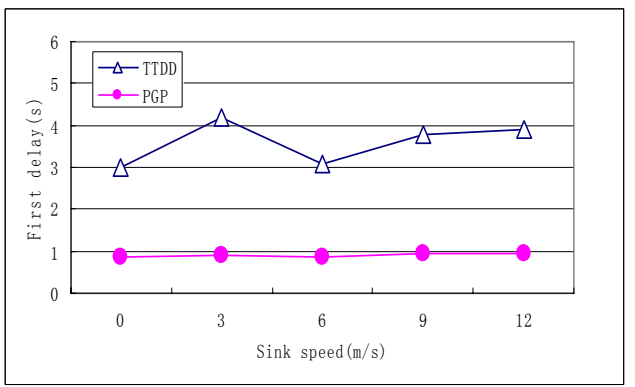

Figure 10. First delay with different speeds.

\subsubsection{Number of sources}

We next study the impact of the number of sources on PGP performance. In the default simulation setting of 100 nodes, sink keeps moving at the speed of $3 \mathrm{~m} / \mathrm{s}$. The numbers of sources we used are 1, 2, 3 and 4 separately. For the first delay, we use the average first delay of the sink-source pairs.

According to the simulation results, the performances of protocols decline with the increment of source number. Let's interpret these parameters separately.

Figure 11 shows the different infection of success rate between PGP and TTDD as the number of source nodes increases. success 
rate of TTDD is higher than that of PGP and the more source nodes in the netrok, the better performance PGP will get.

Figure 12 shows the comparasion with the averange delay through PGP and TTDD. Also, the difference is not so clear when the nmber of source nodes is only one in the network. But as the number of nodes increase, the averanfe delay of PGP is nuch lower than TTDD.

We can see from figure 13 that there are obvious improvement of the first delay through PGP than TTDD.

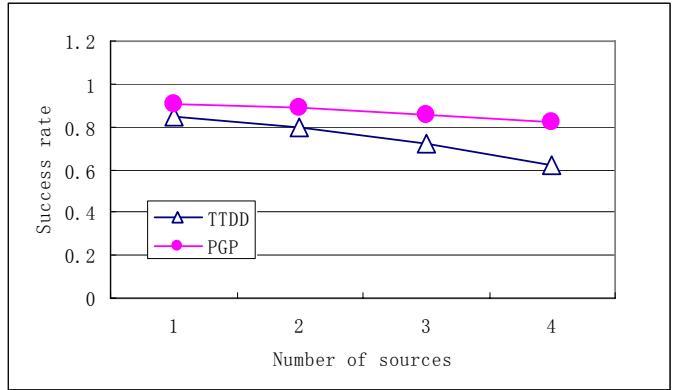

Figure 11. Success rate with different source number.

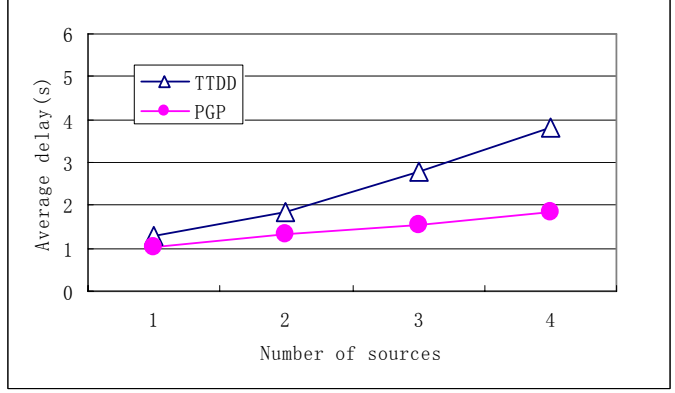

Figure 12. Average delay with different source number.

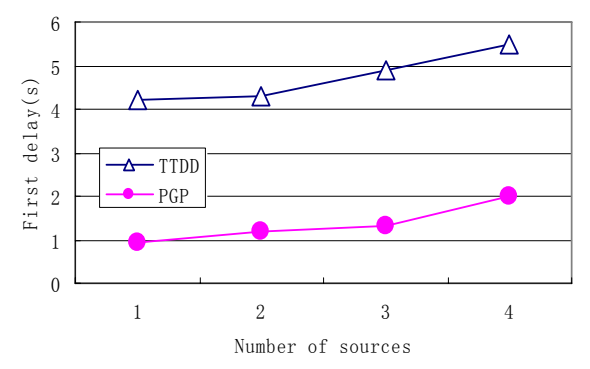

Figure 13. First delay with different source number.

\section{CONCLUSION}

In this paper we present a sink mobility supported route protocol, PGP. It is applicable for environmental monitoring that requires some urgent request. A two-tier grid is constructed at first: a bottom grid with size $\alpha$ and a top grid with $2 \alpha$. Based on this structure, we propose the hierarchical-monitoring mechanism, including coarsely granular monitoring and finely granular monitoring. Each of the top grid points starts coarsely granular monitoring within a $2 \alpha \times 2 \alpha$ square. Once the interest happens, the finely granular monitoring is actuated by the GP1 which detects the interest and its eight neighbor GP0s. The finely granular monitoring is very useful for the condition judgment and trend forecast.

In PGP, only the GP1s can promote themselves as ES and all event sources share the same transmission grid. Therefore the cost of PGP grid construction is less then TTDD. We add a GA into our protocol. That assures the event data push to sink in time and reduces overhead of query flooding. To get the information of any field anytime, PGP supports query-driven. Lastly, we prove that PGP works better than TTDD from some aspects of the network parameters through simulation results.

In the future, we will mainly focus on how to improve PGP to utilize this protocol to more actual applications. Also we will try to ameliorate the protocol to make it more suitable for the sink mobility with the combination of the prediction to the mobile sink

\section{ACKNOWLEDGMENTS}

This research was supported by Nokia-BUPT Joint Project, China 863 High-Tech Programe (No.2007AA01Z222), Programme Sino-Francais de Recherches Avances(No.PRASI07-01), and key project of Inner Mongolia Natural Science Fund (200711020103).

The authors would like to thank Weiwei Jiao and Yan Wang for several constructive discussions.

\section{REFERENCES}

[1] A. Cerpa, J. Elson, D. Estrin, L. Girod, M. Hamilton and J.Zhao. 2001. Habitat Monitoring: Application Driver for Wireless Communications Technology. ACM SIGCOMM Workshop on Data Communications, Latin America. pp: 2041

[2] Fan Ye, Haiyun Luo, Jerry Cheng, Songwu Lu and Lixia Zhang. 2002. A two-tier data dissemination model for large scale wireless sensor networks. The Eighth ACM International Conference on Mobile Computing and Networking, Atlanta, GA, USA. pp: 585-594.

[3] A. Choksi, R. P. Martin, B. Nath, and R. Pupala, 2002. Mobility Support for Diffusion-based Ad-Hoc Sensor Networks, Technical Report DCS-TR-463.

[4] Young-Bae Ko, and Nitin H. Vaidya. 2000. Location-Aided Routing (LAR) in Mobile Ad Hoc Networks. Wireless Network. Volume 6, Number 4. pp: 307-321.

[5] J. Li, J. Jannotti, D. Couto, D. R. Karger, and R. Morris. 2000. A Scalable Location Service for Geographic Ad Hoc Routing. In Proceedings of the Sixth Annual International Conference on Mobile Computing and Networking (MOBICOM'00). pp: 120-130.

[6] Cheng, C.T., Lemberg, H.L. etc. 2002. SLALoM: A Scalable Location Management Scheme for Large Mobile Ad-hoc Networks. IEEE WCNC2002. pp: 574-578

[7] Stefano Basagni, Imrich Chlamtac, Violet R. Syrotiuk and Barry A. Woodward. 1998. A distance routing effect algorithm for mobility (DREAM). In Proceedings of the 4th 
annual ACM/IEEE international conference on Mobile computing and networking. pp: 76-84

[8] Brad. Karp and H. T. Kung. 2000. GPSR: Greedy perimeter stateless routing for wireless networks. ACM/IEEE Mobicom'00. pp: 243-254

[9] Yih-Chun Hu, Johnson,D.B. and Perriq,A. 2003. SEAD: secure efficient distance vector routing for mobile wireless ad hoc networks. Ad Hoc Networks, Volume 1, Issue 1. pp: $175-192$
[10] Akkaya,K and Younis,M, 2004. Energy-aware routing to a mobile gateway in wireless sensor networks. IEEE Globalcom. pp. 16-21.

[11] Jeff Hornsberger and Gholamali C. Shoja, 2005. Geographic Grid Routing for Wireless Sensor Networks. Networking, Sensing and Control. pp: 484-489

[12] Salman Taherian, Dan O'Keeffe and Jean Bacon. 2004. Event Dissemination in Mobile Wireless Sensor Networks. IEEE International Conference on Mobile Ad-hoc and Sensor Systems. pp: 573-575 